\title{
ARTICLE OPEN \\ Screen printing of silver nanowires: balancing conductivity with transparency while maintaining flexibility and stretchability
}

\author{
Weiwei Li (iD) ${ }^{1}$, Shuai Yang ${ }^{1}$ and Atif Shamim ${ }^{1}$
}

Printing metal nanowires are particularly attractive as compared to conventional coating methods due to the ease of processing, direct patterning, and large-scale fabrication capability. However, it is still challenging to print metal nanowire patterns that simultaneously have high conductivity, high transparency, flexibility, and stretchability. Three steps have been taken in this work to balance the transparency and conductivity of the screen-printed flexible and stretchable silver nanowire films, (1) selection of the ink formulation, (2) optimization of the printing parameters, and (3) posttreatment with a laser. The as-obtained silver nanowire patterns are large-area and demonstrate an ultralow sheet resistance of $1.9 \mathrm{ohm} / \mathrm{sq}$, high transmittance (73\%) at the wavelength of $550 \mathrm{~nm}$, and an ultrahigh figure of merit ( 136) as compared to the printed silver nanowire electrodes in the literature. The screenprinted transparent patterns exhibit excellent electrical stability and mechanical repeatability when subjected to 1000 bending cycles with a bending radius of $28 \mathrm{~mm}$ and 1000 stretch-release cycles with $10 \%$ strain, which makes the transparent patterns suitable for the fabrication of flexible, transparent microwave absorbers. The absorption performance of the prepared frequency selective surface absorbers indicates no obvious degradation after various manipulating configurations and multiple bending and stretching cycles. The results are promising enough to make this ink and screen-printing process suitable for many applications of flexible, stretchable, and transparent electronics.

npj Flexible Electronics (2019)3:13; https://doi.org/10.1038/s41528-019-0057-1

\section{INTRODUCTION}

Flexible and stretchable devices have become crucial for relatively new applications such as flexible sensors and actuators, ${ }^{1-3}$ electronic textiles, ${ }^{4-6}$ deformable displays, ${ }^{7}$ and electromagnetic structures. ${ }^{8,9}$ It is essential in these fields to develop electrodes that can offer decent conductive properties despite enduring multimodal and repeated deformations. ${ }^{10}$ Instead of structurally upgrading conventional materials through instability mechanics, the bottom-up assembly of nanomaterials represents an alternative and particularly promising route. ${ }^{11}$ Devices associated with these methods can intrinsically survive large deformations and still maintain remarkable electrical properties. Advances in this context have led to the development of many nanomaterials, including nanowires (NWs), ${ }^{12,13}$ nanotubes, ${ }^{14-18}$ and graphene. ${ }^{19-23}$ Among these building blocks, silver nanowires (Ag NWs) have attracted extensive attention in the last decade because of the excellent mechanical compliance, such as stretchability and flexibility, which is essential for wearable electrodes. ${ }^{3,13,24,25}$ Moreover, many filmlike electrodes made of $\mathrm{Ag} \mathrm{NWs}$ are capable of achieving both high optical transparency and high electrical conductivity, two critical parameters for many optoelectronic devices, including liquid crystal displays, light emitting diodes (LEDs), solar cells, and touch panels. ${ }^{13,26-29}$

Recently, the fabrication of $\mathrm{Ag} \mathrm{NWs}$ and $\mathrm{Ag} \mathrm{NW}$-based electrodes has made significant progress. Today, the reduction of $\mathrm{Ag}$ nitrate in the presence of polyvinyl pyrrolidone (PVP) and metal seeds in ethylene glycol (EG) is a low-cost, solution-based process to synthesize Ag NWs. The fabrication of flexible Ag NW conductors through coating methods ${ }^{30-32}$ and printing methods, ${ }^{25,33,34}$ with various conductivity and transparency has led to specific applications in LEDs, resistive heaters, thin-film transistors, and solar cells. The figure of merit (FoM) can characterize the trade-off between conductivity and transparency in these reported Ag NW films and other transparent conductors (Fig. 1a) by describing the relationship between the transmittance $(T)$ at the wavelength of $550 \mathrm{~nm}$ and the sheet resistance $\left(R_{\mathrm{sh}}\right)$ through Eq. $1^{35}$.

FoM $=\frac{Z_{0}}{2 R_{\mathrm{sh}}\left(T^{-1 / 2}-1\right)}$,

where $Z_{0}$ is the impedance of free space with a value of $377 \mathrm{ohm}$. For transparent conductors, the lowest sheet resistance and the highest transparency are ideal, which means the highest FoM is the best.

The films fabricated by coating methods have typically shown an ultrahigh FoM (even comparable to conventional tin-doped indium oxide), but have also yielded a relatively high sheet resistance. ${ }^{36}$ In contrast, one-step printing methods, such as inkjet printing, screen printing, gravure printing, additive printing, and direct writing, represent a low-cost, large-area fabrication, direct patterning technique that can deposit $\mathrm{Ag} \mathrm{NW}$ patterns with relatively high conductivity. ${ }^{25}$ However, the high Ag loading and/

${ }^{1}$ IMPACT Lab, Computer, Electrical and Mathematical Sciences and Engineering (CEMSE) Division, King Abdullah University of Science and Technology (KAUST), Thuwal 239556900, Saudi Arabia

Correspondence: Weiwei Li (weiwei.li@kaust.edu.sa)

Received: 7 December 2018 Accepted: 14 June 2019

Published online: 10 July 2019 

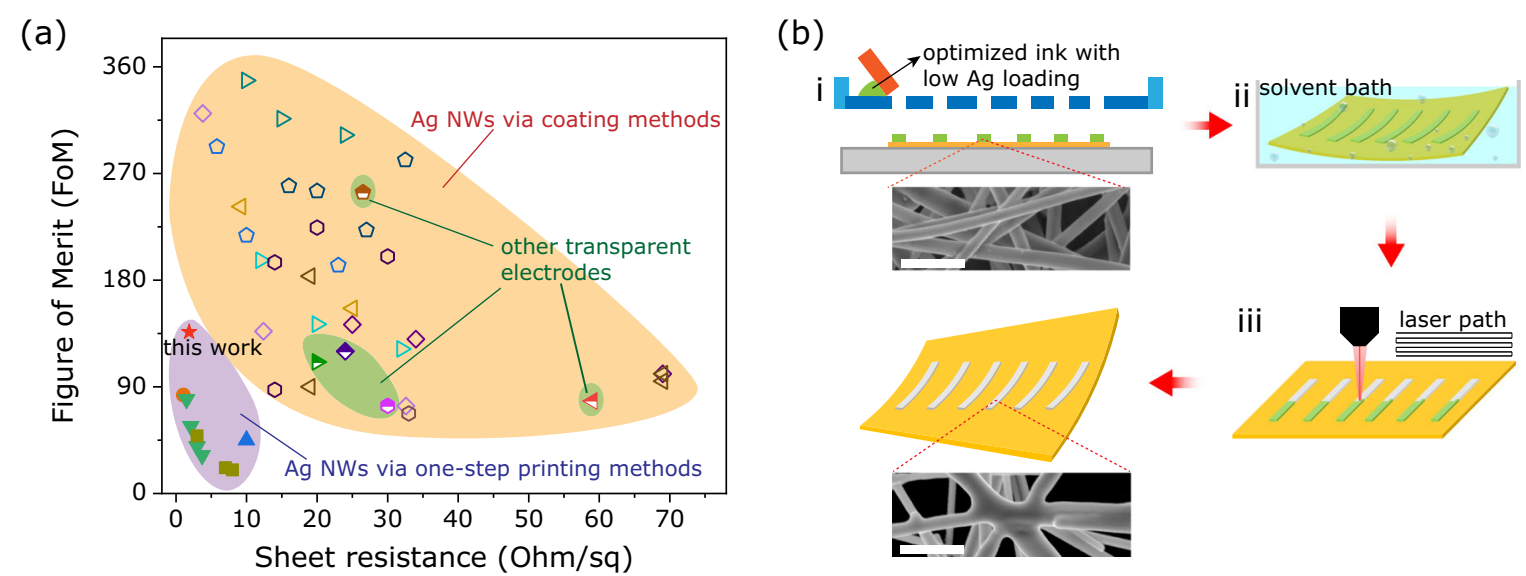

Fig. 1 a Plotting of the FoM as a function of the sheet resistance of the conductive Ag NW films via various coating methods (ground color of yellow) (filtration, ${ }^{8,57}$ drop coating, ${ }^{30}$ spin coating, ${ }^{32}$ spray coating, ${ }^{43}$ and Mayer rod coating ${ }^{13,31}$ ) and one-step printing methods (ground color of purple) (electrohydrodynamic printing, ${ }^{49}$ additive printing, ${ }^{58}$ screen printing, ${ }^{25}$ inkjet printing, ${ }^{34}$ and seed particle printing $\left.{ }^{59}\right)$, as well as some other transparent conductors (ground color of green) through direct writing of silver nanoparticles (Ag NPs), ${ }^{60}$ copper random network, ${ }^{61}$ direct printing of Ag precursors, ${ }^{50}$ and laser transferring. ${ }^{51}$ Note: For the printed Ag NW patterns in the literature, where the transmittance values at the wavelength of $550 \mathrm{~nm}$ are not clearly mentioned, the FoMs have been estimated based on the film thicknesses and the physical appearance of the patterns in comparison to our work. b Schematics for the process to achieve high transparency and conductivity for the printed Ag NW films in this work. (i) Optimization of ink formulation and printing parameters to tune the transparency of the printed Ag NW films. (ii) Immersion of the printed films in a solvent bath to recover the conductivity. (iii) Treatment with strong laser to further increase the conductivity and transparency. The Ag NWs are loosely contacted after printing as shown in the scanning electron microscope (SEM) image in (i), whereas they are completely connected after laser treatment. The scale bars are $500 \mathrm{~nm}$

or reduced $\mathrm{Ag} \mathrm{NW}$ aspect ratio required in printing techniques typically yield a lower transparency of the printed electrodes, which in turn results in a low FoM. ${ }^{34}$ Therefore, simultaneously achieving high conductivity, high transparency and a high FoM has remained somewhat unobtainable for printed Ag NW patterns (Fig. 1a).

In this work, we demonstrate a new kind of Ag NW ink for screen printing to deposit transparent Ag NW patterns on elastic poly(dimethyl siloxane) (PDMS) substrates and optimize the Ag loading, the printing parameters, and the posttreatment with laser to balance the transparency and conductivity, while maintaining the flexibility and stretchability (Fig. 1b). The resulting Ag NW patterns not only exhibit much higher transparency (transmittance as high as $73 \%$ at the wavelength of $550 \mathrm{~nm}$ ) than those printed Ag NW electrodes in the literature (usually below 15\%) but also demonstrate less of a trade-off in the conductivity $(\sim 1.74 \times$ $10^{5} \mathrm{~S} / \mathrm{m}$ ). We further demonstrate our printing method for the application of flexible frequency selective surface (FSS) microwave absorbers, which are typically realized on rigid substrates. The fabricated flexible and semitransparent FSS absorbers with both broadband and narrowband absorption properties exhibit a robust absorption performance even under multiple cycles of bending (radius of $28 \mathrm{~mm}$ ) and stretching (10\% strain). Our fabrication process is simple and easily extended for the fabrication of other large-area flexible, transparent electronics.

\section{RESULTS}

The Ag NWs for the screen-printing ink were synthesized by reducing Ag nitrate in the presence of PVP in EG (see details in the Experimental section). The method proposed here needs a shorter reaction time $(45 \mathrm{~min})$ compared to most of the previously reported methods, ${ }^{30,37,38}$ and is simple to scale up. These features are beneficial to the preparation of the screen-printing ink because of the huge $\mathrm{Ag}$ NWs consumption during the printing process. Figure 2a shows SEM images of the synthesized Ag NWs. By counting $100 \mathrm{Ag} \mathrm{NWs}$, we find that the Ag NWs have an average diameter of $60 \pm 8 \mathrm{~nm}$ and a mean length of $32 \pm 13 \mu \mathrm{m}$. This high length-to-diameter aspect ratio (greater than 500 ) allows for depositing a dense conductive network with a low Ag NWs loading. To further analyze the structure and crystallinity of the synthesized Ag NWs, we performed X-ray diffraction (XRD) of the Ag NWs on a glass substrate; the resultant XRD pattern is shown in Fig. 2b. Notably, four distinct peaks can be observed in the XRD pattern, which can be assigned to (111), (200), (220), and (311). The XRD patterns further confirmed the face-centered-cubic structure of the as-synthesized $\mathrm{Ag} \mathrm{NWs}$ with high qualities without any other impurities.

The purified Ag NWs were directly used to prepare the screenprinting ink. In this study, the ink vehicle was made by dissolving EC and PVP (weight ratio of 1:1) in terpineol to form a homogenous viscous solution. The EC/PVP solution was then mixed with the purified $\mathrm{Ag} \mathrm{NWs,} \mathrm{followed} \mathrm{by} \mathrm{vigorous} \mathrm{stirring} \mathrm{at}$ $60{ }^{\circ} \mathrm{C}$ to evaporate the acetone and residual ethanol. Finally, a small amount of ethanol (1:4 weight ratio to terpineol) was added to the EC/PVP-terpineol-Ag NW mixture to lower the ink viscosity and surface tension. The formulated ink with ethanol is beneficial for obtaining a uniform Ag layer (see Supplementary Information, Fig. S1). The final Ag NWs ink has a solid loading of $\sim 1.97 \mathrm{wt} \%$, which was confirmed by thermogravimetric analysis (TGA), as shown in Fig. 2c. The EC and PVP binder in ink are about $6.1 \mathrm{wt} \%$ (Fig. 2c), which significantly improve the ink viscosity and rheological behavior. Figure $2 \mathrm{~d}$ shows the measured viscosity of the $\mathrm{Ag} \mathrm{NW}$ ink at different shear rates from 1 to $1000 \mathrm{~s}^{-1}$. A shear thinning behavior appears, and the viscosity of the prepared $\mathrm{Ag}$ NW ink at a shear rate of $10 \mathrm{~s}^{-1}$ is about 3.2 Pa.s, which is suitable for screen printing. ${ }^{39}$ The well-formulated Ag NW ink can be directly applied in screen printing (Fig. 2e). Figure $2 f$ demonstrates the simple manufacturing of $\mathrm{Ag} \mathrm{NW}$ patterns on both rigid glass and flexible poly(ethylene terephthalate), where reliable patterns with various shapes and sizes can be fabricated in a large-area and high-efficiency manner.

To achieve high stretchability, we further demonstrate the printing of the Ag NW ink on PDMS substrates. The pristine PDMS substrate features high surface energy and poor wettability, preventing the uniform spreading of ink drops on its surface. After deposition, the aggregated Ag NWs resulted in the discontinuous patterns on the PDMS surface (Fig. S2). To improve the wettability of the PDMS, we employed ultraviolet/ozone (UVO) to treat the surface of the cured PDMS. The middle and right panels of Fig. S2 
(a)

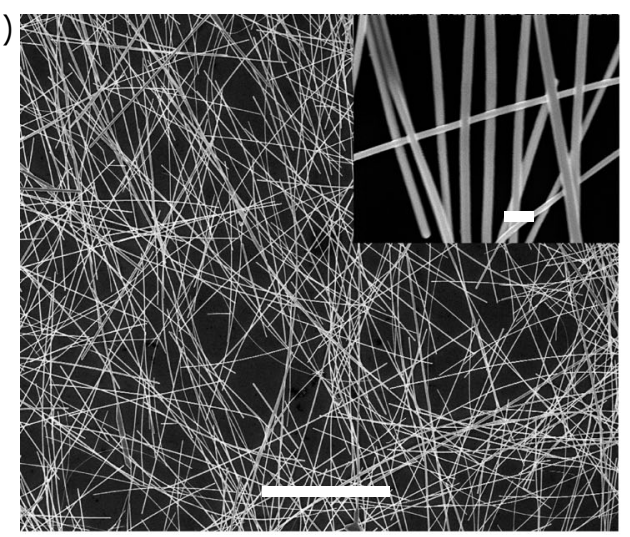

(b)

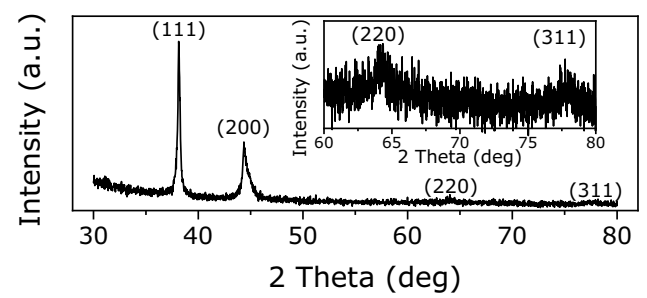

(c)

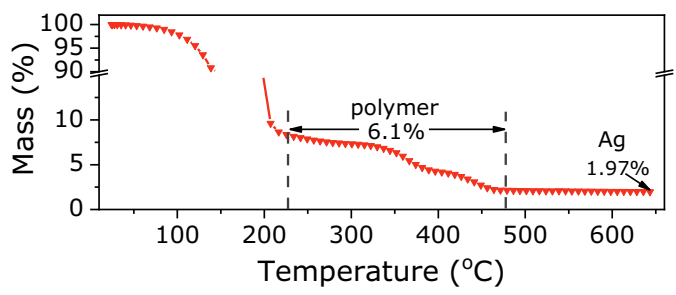

(d)

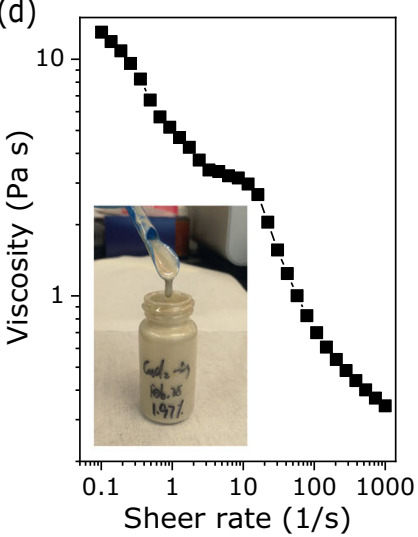

(e)
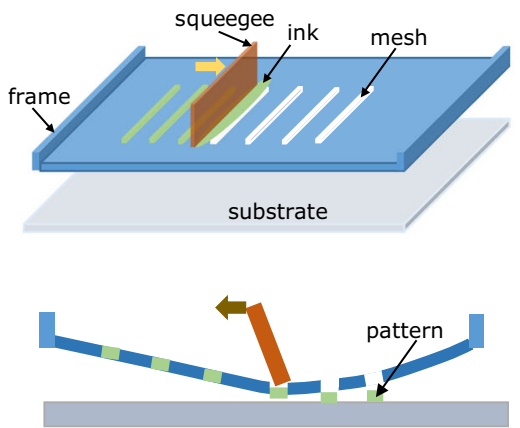

(f)
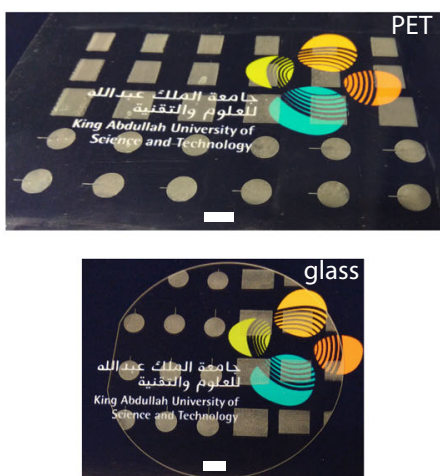

Fig. 2 Properties of the synthesized Ag NWs and the prepared Ag NW ink. a SEM images of synthesized Ag NWs. The scar bar is $10 \mu m$. The inset is a magnified image to highlight the diameter. The scale bar in the inset is $200 \mathrm{~nm}$. b XRD pattern of synthesized Ag NWs. c The thermogravimetric analysis (TGA) of prepared Ag NW screen-printing ink; $6.1 \%$ represents the content of the ethylene cellulose (EC) and PVP in ink and $1.97 \%$ denotes the residue mass, indicating the Ag loading. $\mathbf{d}$ Viscosity at shear rates ranging from 0.1 to $1000 \mathrm{~s}^{-1}$ for the Ag NW ink. The inset is a photograph of the prepared Ag NW ink. e Schematic of the screen-printing process. $f$ The digital images of the screenprinted Ag NW patterns on a flexible poly(ethylene terephthalate) film (top panel) and a rigid glass (bottom panel) with the KAUST logos as the background. The scale bars are $10 \mathrm{~mm}$

are the optical images of the Ag NWs printed on the UVO-treated PDMS; the resulting Ag NW network showed increased uniformity on the PDMS substrate as the UVO-treatment time increased. All the subsequent printings were carried out on PDMS substrates with a $20 \mathrm{~min}$ UVO treatment. Furthermore, similar to the previously reported strategy, ${ }^{25}$ we further applied solvent baths with acetone and ethanol to the printed Ag NW patterns to enhance the conductivity. We have investigated the immersion time for the printed patterns in acetone, and the related sheet resistances are depicted in Fig. 3a. It is observed that the conductivity increases with the immersion time, and 2 min seems to be sufficient to obtain the desired resistance.

Although the sheet resistance of the $\mathrm{Ag} \mathrm{NW}$ networks is significantly reduced after washing (from 460 to $41 \mathrm{ohm} / \mathrm{sq}$ ), it remains unsuitable for applications in some devices, such as flexible LEDs, ${ }^{40}$ solar cells, ${ }^{36}$ and microwave absorbers. ${ }^{41}$ In fact, several posttreatment methods have been introduced in the literature to improve the conductivity of Ag NW networks. Chemical treatments, such as rinsing the $\mathrm{Ag} \mathrm{NW}$ network in solutions or vapors of sodium borohydride, ${ }^{42}$ cetyltrimethylammonium bromide, ${ }^{36}$ sodium halide salts, ${ }^{43}$ hydrogen chloride, ${ }^{44}$ and water moisture, ${ }^{45}$ could induce nanowelding of $\mathrm{Ag} \mathrm{NW}$ junctions and hence reduce the contact resistance between the NWs. However, additional washing procedures are needed that are nontrivial. Recent works have found that conductivity favorable nanowelding can also be realized through simple laser sintering. ${ }^{46,47}$ Herein, a Yb:fiber laser with a power of $9 \mathrm{~W}$ and a scan speed of $150 \mathrm{~mm} / \mathrm{s}$ (corresponding to an exposure time of $\sim 0.67 \mathrm{~ms}$ ) was introduced to sinter the Ag NW network after immersion in acetone for $2 \mathrm{~min}$. As shown in Fig. 3a (green columns), the sheet resistance decreased dramatically after the laser-sintering process. Specifically, the sheet resistance fell from an initial value of $53-7.1 \mathrm{ohm} / \mathrm{sq}$ for samples with $2 \mathrm{~min}$ of immersion and laser sintering. Interestingly, the transmittance of the laser-sintered Ag NW film also improved from 52 to $58 \%$, as illustrated in Fig. 3b. To further explore the finding and balance the transparency and conductivity, we compare a series of screenprinted Ag NWs films with different thicknesses, and in turn various initial transmittance and sheet resistance values have observed. First, Ag NW conductive inks with various Ag loadings from 1 to $7 \%$ were prepared, and the trade-off between the transparency and conductivity of the screen-printed films was studied (Fig. S3). When the Ag loading was increased from 1 to $7 \%$, the transmittance decreased from 69 to $13 \%$, but at the same time, the sheet resistance also decreased from 156 to $21 \mathrm{ohm} / \mathrm{sq}$. Here, the Ag NW ink with a $2 \%$ loading was chosen as a satisfactory compromise between the relatively high transmittance $(53 \%)$ and low sheet resistance $(57 \mathrm{ohm} / \mathrm{sq})$. Second, the transparency and conductivity of the screen-printed Ag NW films were balanced by tuning the printing parameters: the printing 
(a)

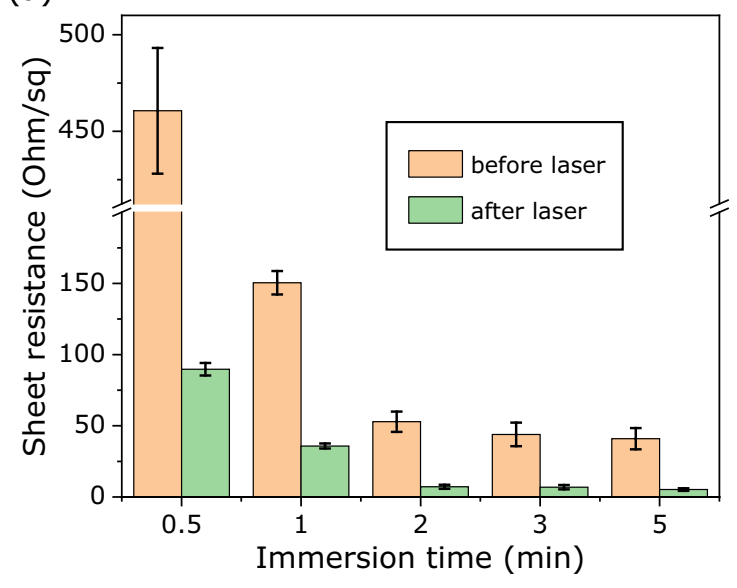

(c)

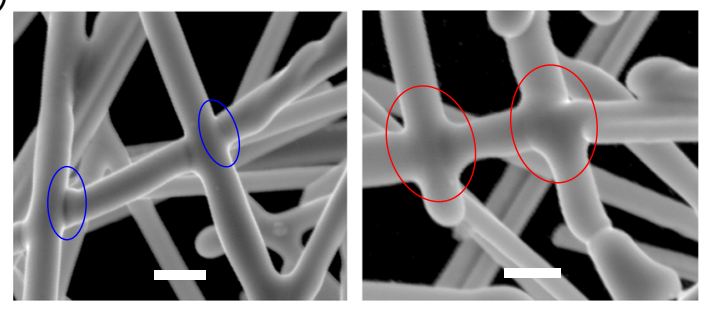

(b)

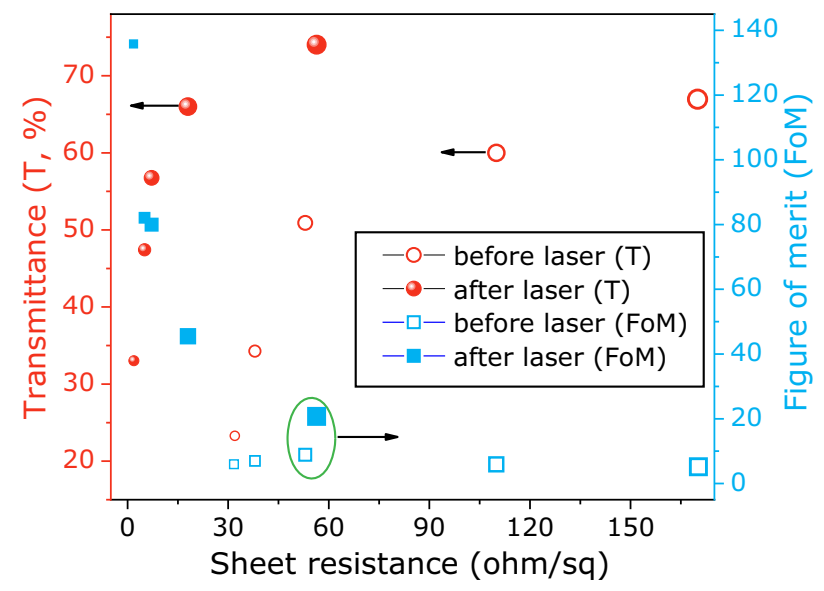

(d)

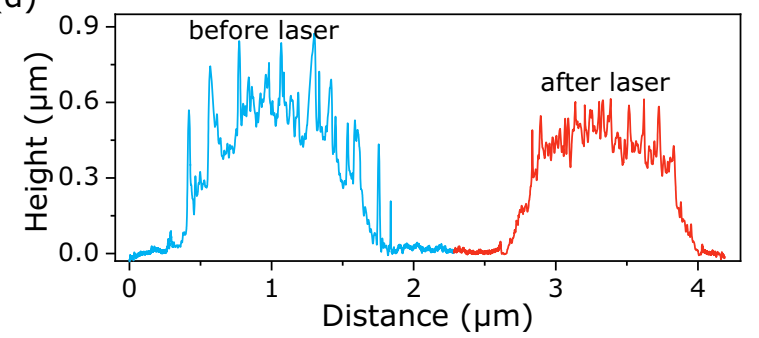

Fig. 3 Properties of the screen-printed and laser-treated Ag NW patterns. a Dependence of the sheet resistance of the fabricated screenprinted Ag NWs after immersion in acetone for various time periods and laser treatment. $\mathbf{b}$ The measured transmittance at the wavelength of $550 \mathrm{~nm}$ (spheres) and the FoM (squares) as a function of the sheet resistance of the screen-printed Ag NW films. The faster printing speed, higher force, smaller gap, and larger number of printing cycles indicate the smaller of the plotted markers. Markers with the same size represent the same sample before (empty) and after (solid) laser treatment. c SEM image of Ag NW junctions after laser treatment. The junctions are welded (blue circles) and even tightly connected (red circles) after laser treatment. The scale bars are $100 \mathrm{~nm}$. $\mathbf{d}$ Profile of the screen-printed Ag NW film before (black line) and after (red line) laser treatment, indicating a slight reduction in the thickness of the Ag NW layer after laser treatment

speed (from $50 \mathrm{~mm} / \mathrm{s}$ to $200 \mathrm{~mm} / \mathrm{s}$ ), the squeeze force, the gap between the mesh and substrate $(1-5 \mathrm{~mm})$, and the number of printing cycles (1-3 cycles). Typically, a faster printing speed (higher than $100 \mathrm{~mm} / \mathrm{s}$ ), higher force, smaller gap (less than $4 \mathrm{~mm}$ ), and larger number of printing cycles (more than one cycle) lead to thicker printed films and, in turn to reduce transparency. The transmittances of these screen-printed Ag NW films varied from 23 to $67 \%$ at $550 \mathrm{~nm}$ (Fig. S3 with solid lines), whereas the sheet resistance increased from 32 to $170 \mathrm{ohm} / \mathrm{sq}$. Finally, the laser system was introduced to further balance the transparency and conductivity. The laser-sintering treatment proved effective in improving the transparency (Fig. 3a and Fig. S4 with dashed lines) and a more than $10 \%$ improvement in transmittance was seen. At the same time, the sheet resistance of the laser-sintered Ag NW patterns was only $\sim 11 \%$ compared to that of the washed patterns (Fig. 3a). These results demonstrate superior transparency compared to the various printed Ag NWs films and some other transparent conductors in the literature (Fig. 1a) and one of the lowest sheet resistances $(1.9 \mathrm{ohm} / \mathrm{sq})$. For comparison's sake, other studies have reported the following sheet resistance and transmittance values: screen-printed Ag NW patterns (calculated sheet resistance, $1.05 \mathrm{ohm} / \mathrm{sq}$; no transparency data), ${ }^{25}$ gravureprinted Ag NW patterns (calculated sheet resistance, $0.468 \mathrm{ohm} /$ sq; no transparency data), ${ }^{48}$ electrohydrodynamic printed Ag NW patterns (sheet resistance, $1.5-3.7 \mathrm{ohm} / \mathrm{sq}$; no transparency data), ${ }^{49}$ direct printing of $\mathrm{Ag}$ precursor (sheet resistance, $27.6 \mathrm{ohm} / \mathrm{sq}$; transmittance: $94.7 \%),{ }^{50}$ and laser transferred Ag NW patterns (sheet resistance, $59.1 \mathrm{ohm} / \mathrm{sq}$; transmittance: $92.3 \%) .{ }^{51}$ To further highlight the influence of laser sintering on the properties of transparent patterns, we calculated and plotted the FoM of the printed Ag NW patterns before and after laser sintering, as illustrated in Fig. 3b. The calculated FoMs of the printed patterns after laser sintering increased to nearly 140 from an initial value of 10 . The results also indicate the effectiveness of laser sintering in terms of improving the transparency and conductivity of the screen-printed Ag NW patterns.

The mechanism through which laser treatment improves the transparency and conductivity was further studied by investigating the detailed morphologies of Ag NWs before and after laser treatment through SEM (Figs 3c and S5). Melted NWs and fused NW junctions were observed after laser sintering (Fig. $3 \mathrm{c}$ and top left inset of Fig. S5). In contrast, the contact between the Ag NWs was relatively loose for the $\mathrm{Ag} \mathrm{NW}$ networks without laser sintering (top right inset of Fig. S5). Thus, we attribute such a dramatic decrease in the sheet resistance to the fusion of the Ag NW junctions. In addition, it is also observed that the laser treatment reduced the thickness of the Ag NW film, as measured in Fig. 3d (decrease of $14 \%$ ), which may lead to improved transparency of this Ag NW network. In fact, the laser sintering was carried out with a fiber laser operating at a $1.06 \mu \mathrm{m}$ wavelength. For Ag, a low transmittance of $30 \%$ was observed within a wavelength of $1.06 \mu \mathrm{m}$, and most of the emitted energy was absorbed, ${ }^{52}$ resulting in the melted $\mathrm{Ag} \mathrm{NW}$ junctions and completely connected Ag NWs (Fig. 3c). As a result, not only was the sheet resistance significantly reduced but the transparency of the Ag NW film also improved because of the decreased thickness.

To demonstrate the potential applications for the screenprinted stretchable electrodes, we have proposed transparent FSS 

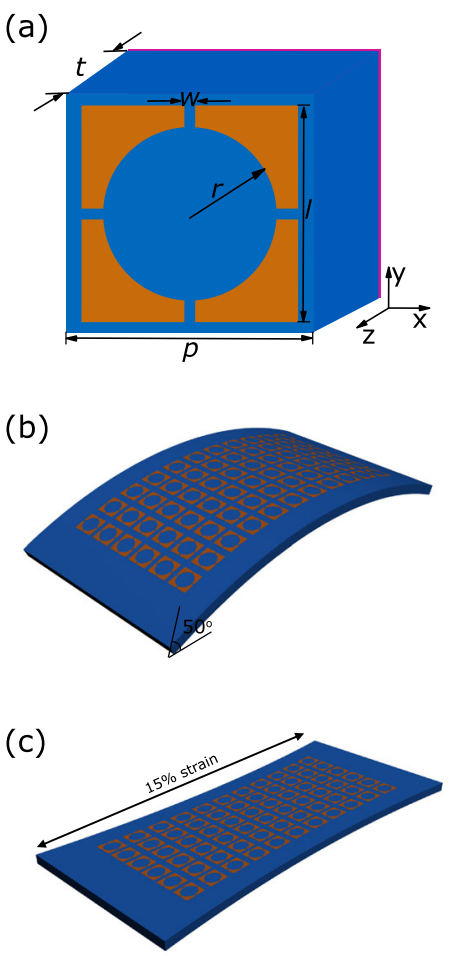
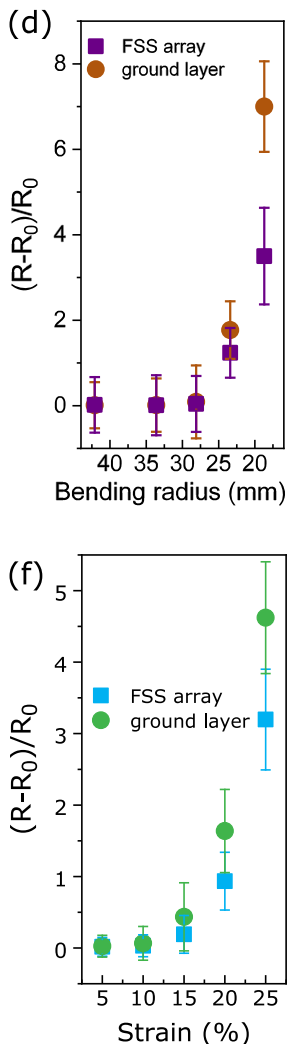
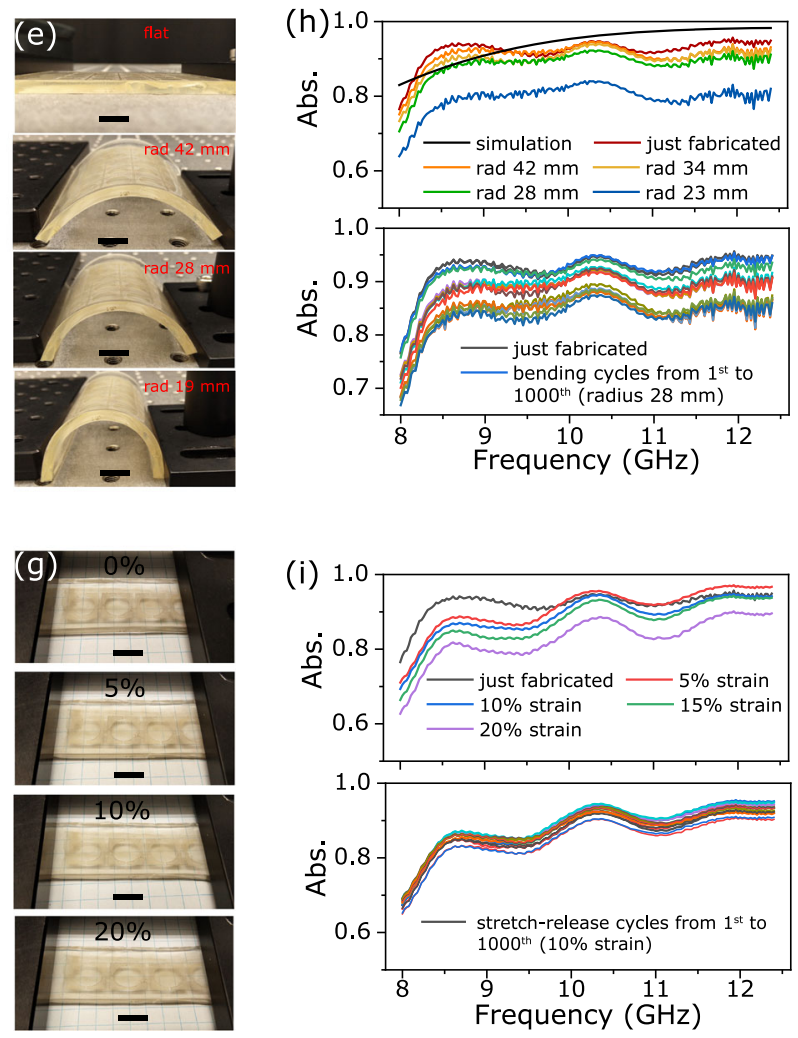

Fig. 4 Properties of the proposed FSS absorbers. a Perspective view of the constructed 3D model of a unit cell of the proposed broadband absorber in CST. The geometry parameters are $p=11 \mathrm{~mm}, I=10 \mathrm{~mm}, r=4 \mathrm{~mm}, w=0.5 \mathrm{~mm}$, and $t=3.5 \mathrm{~mm}$. The dielectric substrate has a permittivity of $2.32+0.005 \mathrm{j}$. The conductivity of the FSS layer (orange) is $200 \mathrm{~S} / \mathrm{m}$, corresponding to a sheet resistance of 50 ohm/sq. The ground layer (pink part) has a conductivity of $2.7 \times 10^{4} \mathrm{~S} / \mathrm{m}$. b Schematic illustration of bending the FSS absorber with a bending angle of $50^{\circ}$. c Schematic illustration of stretching the FSS absorber with 15\% strain. $\mathbf{d}$ The sheet resistance change of the FSS layer and ground layer as a function of bending radiuses ranging from 42 to $19 \mathrm{~mm}$. e Digital images of the FSS absorber bent at different radiuses. The scale bars are $10 \mathrm{~mm}$. $\mathbf{f}$ The resistance change in the FSS layer and ground layer as a function of the tensile strain, which ranged from 5 to $25 \%$. $\mathbf{g}$ Digital images of the FSS absorber stretched under different tensile strains. The scale bars are $10 \mathrm{~mm}$. $\mathbf{h}$ The measured absorptivity of the fabricated absorber under bending radius of 42,34, 28, and $23 \mathrm{~mm}$ (top), and bending cycles from 1 to 1000 with a radius of $28 \mathrm{~mm}$ (bottom). $\mathbf{i}$ The measured absorptivity of the fabricated absorber under various tensile strains of $5,10,15$, and $20 \%$ (top), and stretching cycles when $10 \%$ strain (bottom)

absorbers (Fig. 4a) with broadband and narrowband absorption in this work through the above-mentioned Ag NW screen-printing process. Typically, an FSS absorber consists of a dielectric substrate separated by an FSS layer with a periodic array to optimize the reflection and absorption and a ground layer with high conductivity to minimize the wave transmission. ${ }^{53}$ All three layers should be optically transparent to fabricate a transparent FSS absorber with potential applications in smart transparent windows for radio wave isolation and solar panels to enhance light absorption. ${ }^{41}$ Here, the Ag NW FSS layer was screen printed on UVO-treated PDMS with a thickness of $3.5 \mathrm{~mm}$ (Fig. 4e); immersion in acetone for $2 \mathrm{~min}$ and ethanol for $5 \mathrm{~min}$ (sheet resistance of $51 \pm 2.4 \mathrm{ohm} / \mathrm{sq}$ ) followed. An Ag NW ground layer was printed on the other side of the PDMS without a mask, resulting in a continuous conductive layer. The ground Ag NW layer was treated with a laser to achieve sheet resistance as low as $7.5 \mathrm{ohm} / \mathrm{sq}$ (corresponding to a conductivity of $\sim 1.9 \times 10^{5} \mathrm{~S} / \mathrm{m}$ ) to avoid wave transmitting from the absorber (Fig. S9). We highlight the high flexibility of the fabricated broadband FSS absorber achieved through our procedures by evaluating the mechanical compliance, i.e., bending (Fig. 4b) and stretching (Fig. 4c). The bending stability of the ground layer and printed FSS layer was investigated by bending the fabricated absorber with bending a radius of $42-19 \mathrm{~mm}$ (Fig. 4e), and then the resistance change was recorded. Figure $4 \mathrm{~d}$ shows that there was no obvious change in the electrical resistance after bending the sample with a radius of
$28 \mathrm{~mm}$ for both the ground layer ( 8\%) and FSS layer ( 4\%). The slight difference in the resistance change between the ground layer and FSS layer was attributed to the thicker Ag NW layer of FSS layer than that of the ground layer, resulting in more stable resistance upon deformation. However, the resistance increased dramatically when a smaller bending radius (i.e., 23 and $19 \mathrm{~mm}$ ) was applied to the sample. Microscale cracks can be observed in the SEM images of the Ag NW network after bending to a radius of $23 \mathrm{~mm}$ (Fig. S6a). However, because of the high aspect ratio of the Ag NWs, the cracks remained connected (Fig. S6b), which ensured the conductivity of the entire Ag NW network. Multiple bending cycles were performed, and the resistance remained stable even after 1000 bending cycles with a radius of $28 \mathrm{~mm}$ (Fig. S7a). The stretchability of the screen-printed ground layer and FSS layer was also investigated (Fig. $4 \mathrm{~g}$ ) after evaluating the sheet resistance change under different tensile strains. The resistance of the ground layer and the FSS layer increased by $6 \%$ and $3 \%$, respectively, after applying $10 \%$ tensile strain (Fig. 4f). These characteristics are essential for practical applications of a flexible absorber. Notably, the broadband absorber's application favoring performance can be extended for a narrowband absorber fabricated in a similar way (Figs S7b and S8).

The absorption performance of the fabricated absorbers was studied through the waveguide method (see details in the Experimental section). The simulated absorptivity and measured absorptivity of the screen-printed broadband absorber without 
bending and stretching are shown in Fig. 4h. The simulation and measurement results are highly correlated. Benefiting from the mechanical stability of the screen-printed Ag NWs ground layer and FSS layer, the absorption performances remained stable and did not shrink sharply for a bending radius greater than $28 \mathrm{~mm}$ (Figs $4 \mathrm{~h}$ and S9). Similarly, the measured absorptivity showed only a slight reduction with 5 and $10 \%$ strain (Fig. 4i). It should be noted that the absorptivity exhibited excellent repeatability for more than 1000 bending cycles with bending radius of $28 \mathrm{~mm}$ (Fig. 4h) and 1000 stretch-release cycles when subjected to $10 \%$ strain (Fig. 4i). The fabricated narrowband absorber demonstrated similar flexibility and stretchability, and it withstood a similar bending radius and comparable tensile strain without degradation of the absorption performance (Fig. S9). The excellent stability under mechanical deformations can be ascribed to the inherent robust properties of the $\mathrm{Ag} \mathrm{NWs}$ and the stretchable transparent substrate, which is the limitation of the commonly used indium tin oxide, graphene, or pure metal as the conductive layers and glass or polymethyl methacrylate as the substrates. ${ }^{41,54-56}$ In addition to the mechanical stability, the transparency of the fabricated absorbers was measured and is depicted in Fig. S10. The transmittance of the fabricated broadband absorber was $45 \%$ at the wavelength of $550 \mathrm{~nm}$ (Fig. S10), whereas the narrowband absorber had better transparency, achieving a transmittance of $49 \%$ at the wavelength of $550 \mathrm{~nm}$. The proposed absorbers combine flexibility, stretchability, and transparency, indicating great potential for practical applications of microwave absorbers.

\section{DISCUSSION}

In summary, Ag NWs with a length-to-diameter aspect ratio greater than 500 were synthesized in a high-efficiency and largescale manner, and screen-printed Ag NW ink with a low solid content of $2 \mathrm{wt} \%$ was developed. The trade-off between the conductivity and transparency of the screen-printed Ag NW patterns was well balanced by selecting the correct Ag loading ( $2 \mathrm{wt} \%$ ); optimizing the printing speed (higher than $100 \mathrm{~mm} / \mathrm{s}$ ), squeeze pressure, and number of printing cycles (1-3 cycles); and introducing laser treatment to further increase the conductivity. When screen printed on the surface of UVO-treated PDMS, the Ag NW patterns exhibit a conductivity as high as $1.9 \times 10^{5} \mathrm{~S} / \mathrm{m}$ after removing polymer in chemical solvents and laser sintering. Moreover, a transmittance of $73 \%$ at the wavelength of $550 \mathrm{~nm}$ was achieved, meaning the transparency is superior to that of the printed Ag NW films in the literature. After bending with a radius of $28 \mathrm{~mm}$ and stretching with $10 \%$ strain, no discernible changes in electrical resistance were observed for the screen-printed Ag NW patterns. The absorptivity of the fabricated microwave absorbers exhibits reliable stability and repeatability, and shows no obvious reduction after 1000 cycles of bending with a radius of $28 \mathrm{~mm}$ and 1000 cycles of stretching with $10 \%$ strain.

\section{METHODS}

\section{Ag NW synthesis and ink preparation}

The Ag NWs were synthesized by a modified polyol reduction method with one-pot reaction process. Firstly, 1.2 g PVP was dissolved in EG under vigorous stirring. Then, $1 \mathrm{~g} \mathrm{Ag}$ nitrate was added to the PVP-EG solution. After it was completely dissolved, $4 \mathrm{~g}$ of freshly prepared copper (II) chloride $\left(\mathrm{CuCl}_{2}\right)$-EG solution $(50 \mathrm{mM})$ was quickly injected into the mixed solution and stirred for $1 \mathrm{~min}$. Finally, the mixture solution was transferred into a preheated flask at $130^{\circ} \mathrm{C}$, and the reaction was allowed to proceed for $45 \mathrm{~min}$ to complete the growth of the Ag NWs. The resultant dispersion was washed and purified three times using acetone and ethanol to remove the excess PVP and other chemical residues.

The ink formulation was made by dissolving EC and PVP in terpineol at $90^{\circ} \mathrm{C}$ with the help of magnetic stirring to form a $6 \mathrm{wt} \% \mathrm{EC} / \mathrm{PVP}$-terpineol solution. Then, the ink formulation was added to the purified $\mathrm{Ag} \mathrm{NWs}$, and the mixture was vigorously agitated at $60^{\circ} \mathrm{C}$ for $2 \mathrm{~h}$ to obtain a homogeneous dispersion. Finally, ethanol with a 1:4 weight ratio to terpineol was added to the dispersion and stirred for $10 \mathrm{~min}$ without heating to obtain the final Ag NW ink.

\section{Printing and posttreatment}

First, the PDMS substrate was prepared by mixing PDMS solution and the curing agent (Sylgard 184, Dow Corning) at a 10:1 ratio, followed by degassing in a vacuum oven for $30 \mathrm{~min}$ to remove trapped air bubbles. The PDMS precursor was then poured into two molds with a thickness of 0.8 and $3.5 \mathrm{~mm}$, and cured at $70^{\circ} \mathrm{C}$ for $2 \mathrm{~h}$. To improve the wettability of the PDMS substrate and facilitate the printing of the Ag NW ink, we introduce UVO to treat the surface of the cured PDMS for different time periods. The Ag NW ink was screen printed on the treated PDMS using a screen-printing system (AUREL screen printer 900PA) with a Kapton mask (laser cut from the $\mathrm{CO}_{2}$ laser, Universal Laser System) attached to a blank screen mesh. After printing, the $\mathrm{Ag} \mathrm{NW}$ patterns were heated at $120^{\circ} \mathrm{C}$ for $10 \mathrm{~min}$ in an oven to evaporate the terpineol. The dried $\mathrm{Ag}$ NWs patterns were then immersed in acetone for some time to remove the EC; this step was followed by immersion in ethanol for 5 min to remove the PVP and then naturally dry. A Yb:fiber laser (Universal Laser System) was employed to sinter the solvent-treated Ag NW patterns to further improve their conductivity. The power of the fiber laser was set at $9 \mathrm{~W}$, the scan speed was $150 \mathrm{~mm} / \mathrm{s}$ (corresponding to an exposure time of $\sim 0.67 \mathrm{~ms}$ ), and the $z-$ axis was $5 \mathrm{~mm}$ for the $3.5 \mathrm{~mm}$ PDMS substrate and $2.5 \mathrm{~mm}$ for the $0.8 \mathrm{~mm}$ PDMS substrate.

\section{Simulation and fabrication of the FSS absorbers}

The FSS absorbers comprise three parts: the FSS layer, dielectric substrates, and ground layer. A full wave simulation was carried out through CST Microwave Studio 2017. The periodical boundary condition was set along the $x$ - and $y$-axes, and the propagation of the electromagnetic wave was along the $z$-axis. The conductivity of the FSS layer was set at $6.6 \times 10^{3} \mathrm{~S} / \mathrm{m}$, and $2 \times 10^{5} \mathrm{~S} / \mathrm{m}$ for the broadband and narrowband absorbers, respectively. The dielectric substrate has a permittivity of $2.32 \pm 0.005 j$, and the thicknesses are 3.5 and $0.8 \mathrm{~mm}$ for broadband and narrowband absorbers, respectively. A 3- $\mu \mathrm{m}$-thick ground layer with a conductivity of $6.7 \times 10^{4} \mathrm{~S}$ / $\mathrm{m}$ was placed on the back side of the unit cell. The frequency range is 8-12.4 GHz (X-band). For the fabrication of the broadband FSS absorber, Ag NW ink was printed on 3.5-mm-thick PDMS and immersed in acetone for $2 \mathrm{~min}$. For the narrowband absorber, the Ag NW patterns on 0.8-mmthick PDMS were further sintered by laser, as mentioned above.

\section{Characterizations}

A scanning electron microscope (FEI NovaNano FEG-SEM 630) was used to observe the microstructure and morphology of the synthesized Ag NWs and Ag NW patterns. XRD (Bruker D2 PHASER) was carried out to examine the crystallinity of the Ag NWs. The UV-vis absorption spectrum of the Ag NW film was obtained using a UV-vis spectrophotometer (Thermo Evolution 600) in the range of 360 to $800 \mathrm{~nm}$. The shear viscosity of the Ag NW ink was measured at room temperature using a HAAKE MARS2 Rheometer. TGA curve was obtained using a NETZSCH Instrument TG 209 $\mathrm{F} 1$ under a nitrogen atmosphere and the temperature increases from room temperature to $640^{\circ} \mathrm{C}$ at a heating rate of $20^{\circ} \mathrm{C} / \mathrm{min}$. The sheet resistance of the Ag NWs patterns was determined by a four-probe measurement system (CMT-SR2000N). The bending and stretching of the absorbers were performed manually. The absorption performance was evaluated through the waveguide method. After careful calibration of two waveguides, two unit cells of the absorbers were placed in the center of the waveguide and, S11 and S21 with real and imaginary parts were recorded using an X-band waveguide connected to a vector network analyzer (Agilent N5225A). The absorptivity was calculated by the following equation,

$A(\omega)=1-R(\omega)-T(\omega)=1-|\mathrm{S} 11|^{2}-|\mathrm{S} 21|^{2}$,

where $R(\omega)=|\mathrm{S} 11|^{2}$ is the reflectivity, $T(\omega)=|\mathrm{S} 21|^{2}$ is the transmissivity and $\omega$ is the frequency.

\section{DATA AVAILABILITY}

The datasets generated during and/or analyzed during the current study are available from the corresponding author on reasonable request. 


\section{ACKNOWLEDGEMENTS}

The research reported in this publication was supported by funding from King Abdullah University of Science and Technology (KAUST).

\section{AUTHOR CONTRIBUTIONS}

All authors contributed to the preparation of this manuscript. W.L. designed, fabricated the devices, and analyzed the data. W.L. and S.Y. performed the test. A.S supervised the research and corrected the paper. Special thanks to Liu Wang and Zhaohe Dai from the University of Texas at Austin for fruitful discussion, contributions to the writing of this paper.

\section{ADDITIONAL INFORMATION}

Supplementary information accompanies the paper on the npj Flexible Electronics website (https://doi.org/10.1038/s41528-019-0057-1).

Competing interests: The authors declare no competing interests.

Publisher's note: Springer Nature remains neutral with regard to jurisdictional claims in published maps and institutional affiliations.

\section{REFERENCES}

1. Agarwal, G., Besuchet, N., Audergon, B. \& Paik, J. Stretchable materials for robust soft Actuators towards Assistive Wearable Devices. Sci. Rep. 6, 34224 (2016).

2. Wang, X., Li, J., Song, H., Huang, H. \& Gou, J. Highly stretchable and wearable strain sensor based on printable carbon nanotube layers/polydimethylsiloxane composites with adjustable sensitivity. ACS Appl. Mater. Interfaces. 10, 7371-7380 (2018).

3. Yao, S., Cui, J., Cui, Z. \& Zhu, Y. Soft electrothermal actuators using silver nanowire heaters. Nanoscale. 9, 3797-3805 (2017).

4. Jeerapan, I., Sempionatto, J. R., Pavinatto, A., You, J.-M. \& Wang, J. Stretchable biofuel cells as wearable textile-based self-powered sensors. J. Mater. Chem. 4 18342-18353 (2016).

5. Jinno, H. et al. Stretchable and waterproof elastomer-coated organic photovoltaics for washable electronic textile applications. Nat. Energy. 2, 780-785 (2017).

6. Kwak, S. S. et al. Fully stretchable textile triboelectric nanogenerator with knitted fabric structures. ACS Nano. 11, 10733-10741 (2017).

7. Sekitani, T. et al. Stretchable active-matrix organic light-emitting diode display using printable elastic conductors. Nat. Mater. 8, 494 (2009).

8. Jung, J. et al. Highly stretchable and transparent electromagnetic interference shielding Film based on silver nanowire percolation network for wearable electronics applications. ACS Appl. Mater. Interfaces. 9, 44609-44616 (2017).

9. Zhang, F. et al. Mechanically stretchable and tunable metamaterial absorber. Appl. Phys. Lett. 106, 091907 (2015).

10. Wang, D. et al. Chemical formation of soft metal electrodes for flexible and wearable electronics. Chem. Soc. Rev. 47, 4611-4641 (2018)

11. Han, S. et al. Mechanically reinforced skin-electronics with networked nanocomposite elastomer. Adv. Mater. 28, 10257-10265 (2016).

12. Ding, S. et al. One-step fabrication of stretchable copper nanowire conductors by a fast photonic sintering technique and its application in wearable devices. ACS Appl. Mater. Interfaces. 8, 6190-6199 (2016).

13. Cho, S. et al. Large-area cross-aligned silver nanowire electrodes for flexible, transparent, and force-sensitive mechanochromic touch screens. ACS Nano. 11 4346-4357 (2017).

14. Yamada, T. et al. A stretchable carbon nanotube strain sensor for human-motion detection. Nat. Nanotechnol. 6, 296-301 (2011).

15. Shin, S. R. et al. Aligned carbon nanotube-based flexible gel substrates for engineering bio-hybrid tissue actuators. Adv. Funct. Mater. 25, 4486-4495 (2015).

16. Chortos, A. et al. Mechanically durable and highly stretchable transistors employing carbon nanotube semiconductor and electrodes. Adv. Mater. 28, 4441-4448 (2016).

17. Li, W. et al. A temperature-activated nanocomposite metamaterial absorber with a wide tunability. Nano Res. 11, 3931-3942 (2018)

18. Dai, Z. et al. Three-dimensional sponges with super mechanical stability: harnessing true elasticity of individual carbon nanotubes in macroscopic architectures. Sci. Rep. 6, 18930 (2016)

19. Chen, M. et al. Highly stretchable conductors integrated with a conductive carbon nanotube/graphene network and 3D porous poly(dimethylsiloxane). Adv. Funct. Mater. 24, 7548-7556 (2014).
20. Li, M., Tang, Z., Leng, M. \& Xue, J. Flexible solid-state supercapacitor based on graphene-based hybrid films. Adv. Funct. Mater. 24, 7495-7502 (2014).

21. $\mathrm{Li}, \mathrm{X}$. et al. Large-area ultrathin graphene films by single-step marangoni selfassembly for highly sensitive strain sensing application. Adv. Funct. Mater. 26, 1322-1329 (2016).

22. Dai, Z. et al. Hierarchical graphene-based films with dynamic self-stiffening for biomimetic artificial muscle. Adv. Funct. Mater. 26, 7003-7010 (2016).

23. Dai, Z. et al. Multifunctional polymer-based graphene foams with buckled structure and negative poisson's ratio. Sci. Rep. 6, 32989 (2016).

24. Choi, S. J., Kim, S. J., Jang, J. S., Lee, J. H. \& Kim, I. D. Silver Nanowire embedded colorless polyimide heater for wearable chemical sensors: improved reversible reaction kinetics of optically reduced graphene oxide. Small. 12, 5826-5835 (2016).

25. Liang, J., Tong, K. \& Pei, Q. A water-based silver-nanowire screen-print ink for the fabrication of stretchable conductors and wearable thin-film transistors. Adv. Mater. 28, 5986-5996 (2016).

26. Kim, D.-Y., Han, Y. C., Kim, H. C., Jeong, E. G. \& Choi, K. C. Highly transparent and flexible organic light-emitting diodes with structure optimized for anode/cathode multilayer electrodes. Adv. Funct. Mater. 25, 7145-7153 (2015).

27. Zhao, G. et al. Bendable solar cells from stable, flexible, and transparent conducting electrodes fabricated using a nitrogen-doped ultrathin copper film. Adv. Funct. Mater. 26, 4180-4191 (2016).

28. Kim, C.-C., Lee, H.-H., Oh, K. H. \& Sun, J.-Y. Highly stretchable, transparent ionic touch panel. Science. 353, 682-687 (2016).

29. Wang, J. et al. Rod-coating: towards large-area fabrication of uniform reduced graphene oxide films for flexible touch screens. Adv. Mater. 24, 2874-2878 (2012).

30. Jiu, J. et al. Facile synthesis of very-long silver nanowires for transparent electrodes. J. Mater. Chem. A. 2, 6326-6330 (2014).

31. Li, B., Ye, S., Stewart, I. E., Alvarez, S. \& Wiley, B. J. Synthesis and purification of silver nanowires to make conducting films with a transmittance of $99 \%$. Nano Lett. 15, 6722-6726 (2015).

32. Zhang, Y., Guo, J., Xu, D., Sun, Y. \& Yan, F. One-pot synthesis and purification of ultralong silver nanowires for flexible transparent conductive electrodes. ACS Appl. Mater. Interfaces. 9, 25465-25473 (2017).

33. Yao, S. \& Zhu, Y. Wearable multifunctional sensors using printed stretchable conductors made of silver nanowires. Nanoscale. 6, 2345-2352 (2014).

34. Finn, D. J., Lotya, M. \& Coleman, J. N. Inkjet printing of silver nanowire networks. ACS Appl. Mater. Interfaces. 7, 9254-9261 (2015).

35. Lee, $C$. et al. Flash-induced nanowelding of silver nanowire networks for transparent stretchable electrochromic devices. Sci. Rep. 8, 2763 (2018).

36. $\mathrm{Xu}, \mathrm{F}$. et al. Preparation and cold welding of silver nanowire based transparent electrodes with optical transmittances $>90 \%$ and sheet resistances $<10 \mathrm{ohm} / \mathrm{sq}$ J. Colloid Interface Sci. 512, 208-218 (2018).

37. Tokuno, T. et al. Fabrication of silver nanowire transparent electrodes at room temperature. Nano Res. 4, 1215-1222 (2011)

38. Lu, H.-Y., Chou, C.-Y., Wu, J.-H., Lin, J.-J. \& Liou, G.-S. Highly transparent and flexible polyimide-AgNW hybrid electrodes with excellent thermal stability for electrochromic applications and defogging devices. J. Mater. Chem. C. 3, 3629-3635 (2015).

39. Hyun, W. J., Secor, E. B., Hersam, M. C., Frisbie, C. D. \& Francis, L. F. High-resolution patterning of graphene by screen printing with a silicon stencil for highly flexible printed electronics. Adv. Mater. 27, 109-115 (2015).

40. Ricciardulli, A. G., Yang, S., Wetzelaer, G.-J. A. H., Feng, X. \& Blom, P. W. M. Hybrid silver nanowire and graphene-based solution-processed transparent electrode for organic optoelectronics. Adv. Funct. Mater. 28, 1-6 (2018).

41. Jang, T., Youn, H., Shin, Y. J. \& Guo, L. J. Transparent and flexible polarizationindependent microwave broadband absorber. ACS Photonics. 1, 279-284 (2014).

42. Ge, Y. et al. Direct room temperature welding and chemical protection of silver nanowire thin films for high performance transparent conductors. J. Am. Chem. Soc. 140, 193-199 (2018).

43. Kang, H., Kim, Y., Cheon, S., Yi, G. R. \& Cho, J. H. Halide welding for silver nanowire network electrode. ACS Appl. Mater. Interfaces. 9, 30779-30785 (2017).

44. Liang, $X$. et al. Room-temperature nanowelding of a silver nanowire network triggered by hydrogen chloride vapor for flexible transparent conductive films. ACS Appl. Mater. Interfaces. 9, 40857-40867 (2017).

45. Liu, Y. et al. Capillary-force-induced cold welding in silver-nanowire-based flexible transparent electrodes. Nano Lett. 17, 1090-1096 (2017).

46. Jiu, J. et al. High-intensity pulse light sintering of silver nanowire transparent films on polymer substrates: the effect of the thermal properties of substrates on the performance of silver films. Nanoscale. 5, 11820-11828 (2013).

47. Oh, H. \& Lee, M. Laser-direct fabrication of invisible Ag nanowire electrode pattern on flexible plastic substrate. Thin Solid Films. 636, 375-383 (2017).

48. Huang, Q. \& Zhu, Y. Gravure printing of water-based silver nanowire ink on plastic substrate for flexible electronics. Sci. Rep. 8, 15167 (2018). 
49. Cui, Z., Han, Y., Huang, Q., Dong, J. \& Zhu, Y. Electrohydrodynamic printing of silver nanowires for flexible and stretchable electronics. Nanoscale. 10, 6806-6811 (2018)

50. Lee, Y. et al. Versatile metal nanowiring platform for large-scale nano- and optoelectronic devices. Adv. Mater. 28, 9109-9116 (2016).

51. Zacharatos, F., Karvounis, P., Theodorakos, I., Hatziapostolou, A. \& Zergioti, I. Single step laser transfer and laser curing of ag nanowires: a digital process for the fabrication of flexible and transparent microelectrodes. Materials. 11, 1036 (2018).

52. Sowade, E. et al. Roll-to-roll infrared (IR) drying and sintering of an inkjet-printed silver nanoparticle ink within 1 second. J. Mater. Chem. C. 3, 11815-11826 (2015).

53. Rui Zhang, G. et al. Analysis and design of triple-band high-impedance surface absorber with periodic diversified impedance. J. Appl. Phys. 114, 164103 (2013).

54. Yi, D., Wei, X. \& Xu, Y. Transparent microwave absorber based on patterned graphene: design, measurement, and enhancement. IEEE Trans. Nanotechnol. 16, 484-490 (2017).

55. Sheokand, H. et al. Transparent broadband metamaterial absorber based on resistive films. J. Appl. Phys. 122, 105105 (2017).

56. Shen, Y. et al. Transparent broadband metamaterial absorber enhanced by watersubstrate incorporation. Opt. Express. 26, 15665-15674 (2018).

57. Lee, J. et al. Very long $\mathrm{Ag}$ nanowire synthesis and its application in a highly transparent, conductive and flexible metal electrode touch panel. Nanoscale. 4, 6408-6414 (2012).

58. Le, C. et al. Direct printing for additive patterning of silver nanowires for stretchable sensor and display applications. Adv. Mater. Technol. 3, 1700232 (2018).
59. Layani-Tzadka, M. E., Tirosh, E. \& Markovich, G. Patterning metal nanowire-based transparent electrodes by seed particle printing. ACS Omega. 2, 7584-7592 (2017).

60. Hong, S. et al. Nonvacuum, maskless fabrication of a flexible metal grid transparent conductor by low-temperature selective laser sintering of nanoparticle ink. ACS Nano. 7, 5024-5031 (2013).

61. Suh, Y. D. et al. Maskless fabrication of highly robust, flexible transparent $\mathrm{cu}$ conductor by random crack network assisted cu nanoparticle patterning and laser sintering. Adv. Electron. Mater. 2, 1-7 (2016).

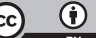

Open Access This article is licensed under a Creative Commons Attribution 4.0 International License, which permits use, sharing, adaptation, distribution and reproduction in any medium or format, as long as you give appropriate credit to the original author(s) and the source, provide a link to the Creative Commons license, and indicate if changes were made. The images or other third party material in this article are included in the article's Creative Commons license, unless indicated otherwise in a credit line to the material. If material is not included in the article's Creative Commons license and your intended use is not permitted by statutory regulation or exceeds the permitted use, you will need to obtain permission directly from the copyright holder. To view a copy of this license, visit http://creativecommons. org/licenses/by/4.0/.

c The Author(s) 2019 